\title{
Advanced Practice Provider Onboarding Support Model Comparison: Is there a Superior Choice?
}

\author{
Melissa Ricker \\ Atrium Health, melissa.ricker@atriumhealth.org \\ Cragin Greene \\ Atrium Health, cragin.greene@atriumhealth.org \\ Anne Vail \\ Atrium Health, anne.vail@atriumhealth.org
}

Follow this and additional works at: https://nsuworks.nova.edu/ijahsp

Part of the Interprofessional Education Commons

\section{Recommended Citation}

Ricker M, Greene C, Vail A. Advanced Practice Provider Onboarding Support Model Comparison: Is there a Superior Choice?. The Internet Journal of Allied Health Sciences and Practice. 2021 Jan 01;19(3), Article 11.

This Literature Review is brought to you for free and open access by the College of Health Care Sciences at NSUWorks. It has been accepted for inclusion in Internet Journal of Allied Health Sciences and Practice by an authorized editor of NSUWorks. For more information, please contact nsuworks@nova.edu. 


\title{
Advanced Practice Provider Onboarding Support Model Comparison: Is there a Superior Choice?
}

\begin{abstract}
Health care practices and institutions are providing variable onboarding models to prepare physician assistants (PAs) and nurse practitioners (NPs) for transition into new practice settings. With the evolution of healthcare delivery and increased opportunities for PAs and NPs to specialize in advanced practice environments, there has been a growing demand for an evidenced-based strategy, structure, and appraisal of content within existing onboarding models. The aim of this paper is to compare two onboarding models for Advanced Practice Providers (APPs): postgraduate training programs and traditional institutional transition to practice programs. With expanding numbers of postgraduate training programs offered nationally and increasing numbers of PA and NP graduates seeking out these opportunities calls into question the differences offered through each model. Postgraduate training programs appear to provide a consistent structure of comprehensive curriculum design, interprofessional learning, meaningful institutional engagement, leadership exposure, and support a superior onboarding support model for PAs and NPs.
\end{abstract}

Author Bio(s)
Melissa Ricker, PA-C, is the PA Fellowship Director at Atrium Health in Charlotte, North Carolina.
Cragin Greene, PA-C is the Academic Director at Atrium Health in Charlotte, North Carolina.
Anne Vail, DNP, AGNP-C, is the PA Fellowship Director at Atrium Health in Charlotte, North Carolina.

This literature review is available in Internet Journal of Allied Health Sciences and Practice: https://nsuworks.nova.edu/ijahsp/vol19/iss3/11 


\title{
The Internet Joưnal of Allied Health Sciences and Practice \\ Dedicated to allied health professional practice and education
}

Vol. 19 No. 3 ISSN 1540-580X

\section{Advanced Practice Provider Onboarding Support Model Comparison: Is there a Superior Choice?}

\author{
Melissa Ricker \\ Cragin Greene \\ Anne Vail \\ Atrium Health \\ United States
}

\begin{abstract}
Background: Health care practices and institutions are providing variable onboarding models to prepare physician assistants (PAs) and nurse practitioners (NPs) for transition into new practice settings. With the evolution of healthcare delivery and increased opportunities for PAs and NPs to specialize in advanced practice environments, there has been a growing demand for an evidenced-based strategy, structure, and appraisal of content within existing onboarding models. Purpose: The aim of this paper is to compare two onboarding models for Advanced Practice Providers (APPs): postgraduate training programs and traditional institutional transition to practice programs. With expanding numbers of postgraduate training programs offered nationally and increasing numbers of PA and NP graduates seeking out these opportunities calls into question the differences offered through each model. Conclusion: Postgraduate training programs appear to provide a consistent structure of comprehensive curriculum design, interprofessional learning, meaningful institutional engagement, leadership exposure, and support a superior onboarding support model for PAs and NPs.
\end{abstract}

Keywords: postgraduate training programs, APP, physician assistant, PA, NP, onboarding 


\section{INTRODUCTION}

Health care has become a heavily discussed social, economic, and essential service. Considering the political and economic polarization of its delivery, we are amid a health care revolution. Proposals of healthcare reform, provider shortages, digital acceleration, and institutional acquisition of private practices are leading the drive for innovation to improve the safety, quality, and efficiency of care. With the patient at the center and healthcare providers leading the way, industry standards must swiftly respond to these changes with a robust, system-wide, and cost-effective approach to improve the standards for both the patient and the provider. Many institutions are expanding the utilization of Advanced Practice Providers (APPs) to meet these stated challenges in highly specialized environments and meet the care demands of an aging population.

In 1965, students enrolled in the first PA and NP programs. ${ }^{1}$ Collectively, these professionals are known as APPs. PA and NP training equipped clinicians with foundational knowledge and skills to enter clinical practice. Shortly after the emergence of these two professions, onboarding models were developed to facilitate efficient transition to the nuances of effective clinical practice, team-based care, and complex care coordination..$^{2,3}$ Since 1971, PA postgraduate training programs have emerged as one type of onboarding support model: providing advanced specialty didactic and clinical training, with the use of formal instructional methods to prepared PAs [and NPs] for clinical practice. ${ }^{2}$ This rivals the traditional transition to practice onboarding model unique to each healthcare institution, varying in duration, content, mode of delivery, resources employed, and competency evaluation. ${ }^{4}$ Ambiguity remains for a standardized definition of effective onboarding support models, with minimal data to describe necessary training duration, prevalence, and effectiveness; but recent data suggests all onboarding models should support skill-based training that will immediately be applied, is timely, relevant, and problem-centered. ${ }^{3-5}$

The aim of this paper is to compare two onboarding support models for APPs: postgraduate training programs and traditional institutional onboarding programs, raising the question, is there is a superior onboarding support model?

\section{LITERATURE REVIEW}

The role of the PA and NP has dramatically expanded over the past thirty years. Today, PAs and NPs are practicing with substantial autonomy in virtually every area of medicine and surgery. Barriers to successful transition to practice include heavy clinical and administrative workload, lack of protective time, insufficient staffing, lack of access to peers or clinicians with expert knowledge, and lack of access to educational environments. ${ }^{3,4}$ How to [best] help new graduate PA and NPs make the transition from student to practicing clinician is of interest to employers and new PAs and NPs. ${ }^{4}$ Onboarding literature in healthcare is scant, however, this literature review will summarize key features of these onboarding support program approaches.

The first postgraduate training program was developed in 1971, designed to train the first PAs that were trained in the general medical model to transition to the field of surgery. ${ }^{6}$ Following the success of this initial program, several additional programs were implemented across the country. In 1988, the Association of Postgraduate Physician Assistant Programs (APPAP) was established to support the specialty postgraduate education and clinical experiences, professional development, and scholarly achievements of PAs and to date, operationally collaborates with over 70 postgraduate PA programs. ${ }^{6}$ Similarly, the Association of Postgraduate NP Programs (APGAP) was organized in 2014 and currently has over 80 institutions represented. ${ }^{7}$ Collectively these two organizations support the following:

- Define the role of postgraduate learner.

- Serve as an information center for PAs, NPs, programs, and other healthcare disciplines.

- Assist in development and maintenance of postgraduate curriculum and evaluation.

- Promote the development of interprofessional, interdisciplinary, and leadership education. ${ }^{6}, 7$

The Physician Assistant Education Association (PAEA) also recognizes PA postgraduate training as an optional advanced training onboarding model and twice in the last two decades, has summarized the state and evolution of these programs. First in 2007 and most recently in 2012, a survey instrument was used to assess demographic information of program directors, obtain unique program structure data, numbers of learners enrolled, and identified academic affiliation. ${ }^{2}$ In 2012, a total of 49 postgraduate programs were identified, with a total of 107 learners enrolled, representing less than $2 \%$ of all new-graduate PAs. ${ }^{2}$ Themes common to all registered programs included:

- Program of at least 6 months duration.

- Formal didactic curriculum and on-site clinical training.

- Enrollment of licensed and credentialed PAs. ${ }^{2}$

While several specialties were represented, the following three specialties were most frequently represented: surgery $(37.5 \%)$, critical care (17.7\%), and emergency medicine (17.5\%). ${ }^{2}$ This is significant, because postgraduate training specialties are offered for all specialties, not just those recognized by the Accreditation Review Commission on Education for the Physician Assistant 
(ARC-PA) or National Commission on Certification of Physician Assistants (NCCPA) required rotations. Ninety percent of the postgraduate training programs report enrolling 4 or less learners per year and all report providing diverse clinical and specialty specific didactics, with several requiring academic presentations, written work, or publications. ${ }^{2}$ To standardize and validate the academic rigor, clinical robustness, and academic requirements of PA postgraduate training programs, the ARC-PA created a voluntary accreditation process for postgraduate PA programs in 2007.8,9

Similar studies have been conducted demonstrating NP postgraduate training program structure and elevated opportunities that program completion have afforded. ${ }^{1}$ The cumulative index technique was used to search for relevant articles searching for existing programs, which identified 14 unique NP postgraduation training programs in $2018 .{ }^{1}$ Standards of postgraduate training programs, were consistent across both professional disciplines including the themes of academic rigor and advanced practice clinical training to all registered NP programs. ${ }^{1,10}$ Similarly, The American Nurses Credentialing Center (ANCC) announced the Practice Transition Accreditation Program (PTAP) accreditation standards in 2015. ${ }^{11}$ The Commission on Accreditation for Practice Transition Programs has a prescribed criteria to demonstrate the quality that is demonstrated through this designation. ${ }^{11}$ The current number of ANCC Primary Accredited Organizations is 439, and PTAP includes 194 Programs in 663 Sites. ${ }^{11}$ There are now several accrediting organizations for NP postgraduate training programs.

Lastly, literature assessing perceptions of the efficacy and perceived utility of postgraduate PA programs was limited, but one study demonstrated of those surveyed:

- $100 \%$ reported employment program post-completion and that the program added value to their career.

- $97 \%$ felt the program made them more competitive in the job market.

- $\quad 95 \%$ would recommend a postgraduate training program to others.

- $90 \%$ felt their postgraduate training program prepared them for leadership. ${ }^{9}$

While formal postgraduate training programs remain optional, their focus on dedicated clinical competency mentoring, general and specialty didactics, professional development training, interprofessional engagement, and research/scholarly education has left graduates feeling clinically and professionally prepared.

An alternative to formal postgraduate training programs is the transition to practice onboarding model. Highlighted in the series, Emerging Practices in Onboarding for PAs, this work supported the necessity of a structured onboarding program, especially for new graduates transitioning to hospital-based settings and community health centers. ${ }^{3,5} \ln 2018$, thirteen PA administrators were interviewed on aspects of institutional onboarding programs and themes surrounding administrative factors necessary for program development; the suggested program content, delivery timeframe, and the strategy were published in a 3-part series. ${ }^{3-5}$ Healthcare administrators unanimously reported an interest in creating structured and reproducible transition to practice program, yet to date, no standardized resource exists. ${ }^{4}$ The five administrative themes suggested for institutional onboarding program design and development included identifying the following:

- Goals of onboarding.

- Critical organizational factors.

- Organizational fit.

- Design.

- Costs. $^{4}$

The administrators were also surveyed on suggested content and topic areas of education to be considered in standardized institutional onboarding programs. The eight program content areas included:

- Culture and values.

- Institutional goals and strategies.

- Task proficiency.

- Rules and policies.

- Structure.

- Working relationships.

- Wellness.

- Institutional navigation and politics. ${ }^{3}$

Lastly, the administrators discussed various strategies used to impart information and for skillset development to sustainably support a transition to practice onboarding program. The following seven strategies were identified:

- Clinical mentoring. 
- Personal and professional mentoring.

- Checking in by administration.

- Shadowing other professionals.

- Delivering didactic content.

- Tailoring content.

- Assessing competency. ${ }^{5}$

While the study series did not demonstrate the validity or reproducibility of these programs across individual institutions, the 13 surveyed reported a hypothesis, by taking the time to onboard clinicians slowly and purposefully, they can improve overall satisfaction among new hires, and reduce costly burnout and costly workforce turnover. ${ }^{3}$ Based on existing literature, each approach provides structured onboarding and mentoring while the new clinician gradually takes on increasing patient responsibilities. ${ }^{1-2,7-9}$ Table 1.0 summarizes key components of postgraduate training programs and traditional institutional transition to practice programs. ${ }^{2-5,9}$

Table 1. Key Components

\begin{tabular}{|l|l|}
\hline Postgraduate Training Program & Traditional Institutional Programs \\
\hline Institution, Fellowship and Specialty Orientation & Institution and Specialty Orientation \\
\hline Pre-determined, 6-18 Month Program Duration & Variable, short-duration Orientation \\
\hline Structured and Comprehensive Curriculum & Tasks Required to Complete Orientation \\
\hline Specialty Specific Didactics & Specialty Specific Rules and Policies \\
\hline Professional Development Education & System Policies and Resources \\
\hline Research/QI Project & Clinical Resource Awareness \\
\hline Interprofessional Education & Working Relationships with Teammates \\
\hline Clinical Supervision for the Duration of the Program & Orientation Mentor \\
\hline
\end{tabular}

\section{DISCUSSION}

Despite their adequate preparation for practice, a growing workforce of APPs entering highly specialized environments, demands a thorough review of the design, content, structure, and value demonstrated for the two most common types of onboarding programs: postgraduate training programs and transition to practice programs. All literature reviewed supported the necessity of onboarding support programs, especially for new graduates transitioning to hospital-based settings and community health centers.

The three common characteristics unique to all PA postgraduate training programs included duration of six months or greater, formal didactic curriculum, and clinical training. ${ }^{2}$ This hybrid training model takes a multimodal approach preparing clinicians to face evolving complexities of healthcare. Through integrating specialty didactics with clinical immersion, those enrolled can longitudinally learn through an established curriculum. Research education and professional development prepare these professionals for meaningful institutional engagement and leadership and interprofessional education and grand rounds provide exposure and appreciation for other health professions. While the value of postgraduate education has been loosely demonstrated by a single study, this comprehensive approach to onboarding has great potential to prepare PAs and NPs to be a leader through the emerging healthcare industry changes. Expansion of scope-of-practice laws for PAs and NPs, such as full practice authority for NPs and optimal team practice for PAs, may heighten the need for effective and efficient onboarding as well. ${ }^{4}$

To help off-set the costs of postgraduate training programs, PAs and NPs enrolled in programs will be hired at a reduced salary or stipend. ${ }^{2}$ Transition to practice programs recommended evaluation of program cost as one of the recommended administrative components. The costs associated with onboarding are due to reduced productivity of the new hire, program leader, and mentors and resources used to implement the curriculum. ${ }^{4}$ While time to peak productivity after hire was not independently investigated, most administrators reported PAs were expected to have a full schedule at $20-40$ weeks after hire. ${ }^{4}$ None of the literature reviewed addressed the specific resources or costs required to implement either of these programs or the financial support required to sustain these programs.

The professional organizations APPAP, APGAP, PAEA, ANCC (PTAP) and ARC-PA recognize PA and NP postgraduate training as a formal, validated, and standardized optional type onboarding program. Developing and existing postgraduate training programs have access to these organizational objective program resources to build, expand, and maintain quality program infrastructure. The multi-pillar structure (didactic, clinical, leadership, and research/quality improvement), quality assurance with institutional alignment, and standard program duration are the three biggest differences between postgraduate training programs 
and transition to practice onboarding programs. ${ }^{2-4} \mathrm{~A}$ single study demonstrated PA postgraduate program graduates perceived their program engagement to high value across the areas of preparedness and satisfaction, while the value in transition to practice models subjectively hypothesized. ${ }^{9}$ However, those questioning the value of postgraduate training, argue it could promote specialization, decreases flexibility, and limits scope of practice.

Postgraduate training program leadership necessitates a minimum of one program director, who can also serve as a clinical and professional mentor. ${ }^{2}$ These programs often utilize several clinical mentors and administrative support staff to provide support to the trainees. Transition to practice program design suggested one physician mentor be assigned to the newly hired PA or NP. This process can be difficult to systematically implement due to time constraints, unique institutional organization, and variability of interaction in team-based care. ${ }^{4}$ While employers strategically and purposefully hire all types of clinicians, postgraduate training programs have the potential to be widely accepted as the superior onboarding support program for PAs and NPs.

\section{CONCLUSION}

Healthcare practices and institutions have supported PAs and NPs entry into practice with various onboarding support models: postgraduate and transition to practice programs. While many institutions feel the investment in onboarding support programs is necessary, widespread uptake of programs will require more research on cost, benefits, and outcomes to add to the current body of evidence. However, based on expanding numbers of postgraduate programs nationally, increased number of PAs and NPs seeking optional postgraduate programs, national postgraduate accreditation standards, national postgraduate advocacy organization resources, and post-program completion perception of high value; to date, postgraduate training programs appear to provide a comprehensive, consistent, and superior onboarding program for PAs and NPs.

\section{References}

1. Sanchez CAC. Current status of fellowship programs for advanced practice registered nurses in the nurse practitioner role. Nurse Educator. 2018;43 (1):42-44.

2. Polansky M. Postgraduate clinical education of physician assistants. J Physician Assistant Educ. 2012;23(1):39-45.

3. Sanchez M, Anglin L, Rana R, Butterfield R, Everett CM, Morgan P. Emerging practices in onboarding programs for PAs: Program content. JAAPA. 2020;33(9):38-42. doi:10.1097/01.JAA.0000694968.61482.

4. Anglin L, Sanchez M, Butterfield R, Rana R, Everett CM, Morgan P. Emerging practices in onboarding programs for PAs: Strategies for onboarding. JAAPA. 2021;34(1):32-38. doi:10.1097/01.JAA.0000723932.21395.74Association of Postgraduate PA Programs. https://appap.org/ Accessed October 18, 2020.

5. Association of Post Graduate APRN Programs. https://apgap.enpnetwork.com Accessed March 4, 2021.

6. Hussaini, Sobia. Accreditation and implications of clinical postgraduate pa training programs. JAAPA. May 2016;29(5):1-7. doi: 10.1097/01.JAA.0000482298.17821.fb

7. Will, K. Perceived efficacy and utility of postgraduate physician assistant training programs. JAAPA. Mar 2016;29(3):46-48. doi: 10.1097/01.JAA.0000480569.39885.c8

8. Alencar, M. Nurse practitioner fellowship. CJON. 2018;22 (2):142-145.

9. American Nurses Credentialing Center. https://www.nursingworld.org/organizational-programs/accreditation/ptap/. Accessed March 15, 2021. 